\title{
Research
}

\section{Opportunistic screening versus usual care for diagnosing atrial fibrillation in general practice:}

\author{
a cluster randomised controlled trial
}

\begin{abstract}
\section{Background}

Atrial fibrillation (AF) increases the risk of stroke, heart failure, and all-cause mortality. AF may be asymptomatic and therefore remain undiagnosed. Devices such as single-lead electrocardiographs (ECGs) may help GPs to diagnose AF
\end{abstract}

\section{Aim}

To investigate the yield of opportunistic screening for AF in usual primary care using a single-lead ECG device.

\section{Design and setting}

A clustered, randomised controlled trial among patients aged $\geq 65$ years with no recorded AF status in the Netherlands from October 2014 to March 2016.

\section{Method}

Fifteen intervention general practices used a single-lead ECG device at their discretion and 16 control practices offered usual care. The followup period was 1 year, and the primary outcome was the proportion of newly diagnosed cases of $A F$

\section{Results}

In total, 17107 older people with no recorded AF status were eligible to participate in the study. In the intervention arm, $10.7 \%$ of eligible patients $(n=919)$ were screened over the duration of the study year. The rate of newly diagnosed AF was similar in the intervention and control practices $(1.43 \%$ versus $1.37 \%, P=0.73)$. Screened patients were more likely to have comorbidities, such as hypertension (60.0\% versus $48.7 \%$ ), type 2 diabetes $(24.3 \%$ versus $18.6 \%)$, and chronic obstructive pulmonary disease $111.3 \%$ versus $7.4 \%$ ), than eligible patients not screened in the intervention arm. Among patients with newly diagnosed AF in intervention practices, $27 \%$ were detected by screening, $23 \%$ by usual primary care, and $50 \%$ by a medical specialist or after stroke/transient ischaemic attack.

\section{Conclusion}

Opportunistic screening with a single-lead ECG at the discretion of the GP did not result in a higher yield of newly detected cases of $A F$ in patients aged $\geq 65$ years in the community than usual care. For higher participation rates in future studies, more rigorous screening methods are needed.

\section{Keywords}

atrial fibrillation; diagnosis; electrocardiograph;

older people; pulse; screening.

\section{INTRODUCTION}

Atrial fibrillation (AF) is a common heart rhythm disorder in older people, which is associated with an increased risk of ischaemic stroke, heart failure, and mortality. ${ }^{1-3}$ Oral anticoagulants can reduce the risk of stroke by approximately $60 \%$ and risk of mortality by approximately $25 \%$ in patients with AF. ${ }^{4} \mathrm{AF}$ is asymptomatic in around $30 \%$ of patients. ${ }^{5}$ Current guidelines recommend opportunistic screening for AF in primary care using pulse palpation or rhythm strip, followed by a 12-lead electrocardiograph (ECG) in case of irregularity. ${ }^{6,7}$ Several screening devices have been developed to help detect AF with a single time-point measurement, such as blood pressure monitors with heart rhythm registration, and single-lead ECG devices. ${ }^{8}$ Single-lead ECG devices seem attractive because 1-minute registrations can be downloaded for interpretation by a cardiologist.

Previous studies have shown that screening with single-lead ECG devices resulted in an increased detection of $A F$ compared with usual care, ${ }^{9-12}$ but these studies performed a systematic screening approach, that is, proactively inviting participants to the GP practice, using popups in the GP's computer, or using an additional team of coworkers to screen patients during an influenza vaccination session. $9.10,12,13$ Opportunistic screening

F Kaasenbrood, MD, PhD, epidemiologist and GP trainee; M Hollander, MD, PhD, GP and assistant professor; $\mathbf{S H M}$ de Bruijn, MD, research student; CPE Dolmans, MD, research student; AW Hoes, $\mathrm{MD}, \mathrm{PhD}$, professor in clinical epidemiology; FH Rutten, MD, PhD, GP and professor in general practice, Julius Center for Health Sciences and Primary Care, University Medical Center Utrecht, Utrecht University, Utrecht. RG Tieleman, MD, PhD, cardiologist, Department of Cardiology, Martini Hospital Groningen; assistant professor, Department of Cardiology, University Medical Center Groningen, Groningen. programmes that leave screening for $\mathrm{AF}$ to the discretion of the GP practice are scarce but worth considering because they are easy to implement; however, most of the above studies were performed before the guidelines suggested opportunistic screening using pulse palpation and it is not known whether detection in usual care might have improved since then.

This study aimed to evaluate whether opportunistic screening with a single-lead ECG device at the discretion of general practice healthcare professionals over 1 year, in patients aged $\geq 65$ years who visited the primary care surgery, resulted in a higher AF detection yield than general practices providing usual care.

\section{METHOD}

\section{Design}

A clustered, randomised controlled trial was performed in which 15 general practices had the opportunity of screening for AF with a single-lead ECG device, and 16 general practices provided usual care.

\section{Participants}

Practices were located in rural, suburban, and urban areas in the Netherlands. All patients aged $\geq 65$ years without a history of $\mathrm{AF}$ and enlisted with the participating GP practices were eligible for inclusion. The study was conducted from October 2014

\section{Address for correspondence}

Femke Kaasenbrood, Julius Center for Health Sciences and Primary Care, University Medical Center Utrecht, Utrecht University, Strat 6.131, PO Box 85500, Utrecht 3508 GA, the Netherlands. Email: f.kaasenbrooddumcutrecht.nl Submitted: 12 April 2019; Editor's response: 3 June 2019; final acceptance: 7 August 2019 CBritish Journal of General Practice

This is the full-length article (published online 28 Jan 2020) of an abridged version published in print. Cite this version as: Br J Gen Pract 2020; DOI: https://doi.org/10.3399/bjgp20X708161 


\section{How this fits in}

Atrial fibrillation (AF) increases the risk of stroke and may be asymptomatic. Screening of people aged $\geq 65$ years in the community with a systematic approach has previously resulted in increased detection of AF. The single electrocardiograph device, MyDiagnostick, has good diagnostic accuracy and could be used for AF screening. In this study, opportunistic screening of those aged $\geq 65$ years during usual care seems not to result in a higher yield of AF detection than usual primary care. Detection of AF within usual primary care including pulse palpation seems to have improved in recent years. In a previous UK study (2002), 1.0\% were newly diagnosed with $A F$ in usual primary care in patients aged $\geq 65$ years during 1 year, whereas this was $1.4 \%$ in the present study. If screening for $\mathrm{AF}$ is left to the discretion of a GP, it is mainly patients with high levels of comorbidity who are investigated.

to March 2016, and lasted 1 year in each of the practices.

\section{Intervention and control practices}

Intervention practices were given two to eight MyDiagnostick ECG devices depending on the size of the GP practice. This is a handheld single-lead ECG device that registers lead I for 1 minute and provides an instant light result based on irregularity of the heartbeat. The MyDiagnostick was shown to have a sensitivity of $94 \%$ and $100 \%$ and a specificity of $93 \%$ and $96 \%$ in two diagnostic accuracy studies when compared with the analysis of a simultaneously performed standard 12-lead ECG interpreted by a single cardiologist, in populations with $\mathrm{AF}$ prevalence of $54 \%$ and $28 \%$, respectively. 14,15 Screening in primary care typically results in a low prevalence of AF laround $2-3 \%$ in those aged $\geq 65$ years without a history of AF), ${ }^{9.11}$ and a negative result with a single-lead ECG device may be convincingly considered as sinus rhythm. However, a positive result with such a device needs confirmation with either a 12-lead ECG or visual interpretation of the MyDiagnostick single-lead ECG registration by an experienced GP or cardiologist.

Intervention practices were instructed to screen all people aged $\geq 65$ years without a history of AF when they visited the practice over the duration of the study year. Intervention practices received short training in how to use the screening device and were given examples on how screening with a single-lead ECG device could be organised; however, implementation was left to their discretion and the research team did not encourage screening.

Before screening, all participants in the intervention arm gave written informed consent and completed a questionnaire on symptoms exhibited that possibly relate to (yet are unrecognised as) AF in the previous month. In case of a positive MyDiagnostick result, GPs were instructed to examine the single-lead ECG recording themselves or: have an experienced GP examine it; obtain a 12-lead ECG; send the singlelead ECG (or 12-lead ECG) to a research cardiologist for interpretation. When AF was diagnosed, further management was left to the discretion of the GP. In case of a green light on the ECG device, AF was considered to be absent and there was no further action.

Control practices were briefly informed about the aim of the study without emphasising the diagnosis of AF. ${ }^{6}$ They were not contacted further until the end of the study period.

\section{Main outcome measures}

The main outcome was newly diagnosed AF cases, either screen detected or diagnosed otherwise, as a percentage of the total population aged $\geq 65$ years without a known history of AF at baseline. Patients with atrial flutter were excluded because they have regular rhythms. A single short-lasting occurrence of AF during or directly after cardiac surgery was not considered to be AF because it is most often self-limiting. ${ }^{16,17}$

Eligible patients were retrieved from the practices' electronic databases; they were all patients aged $\geq 65$ years, with unknown AF status, enlisted with the GP. Details of the search terms used to identify AF cases are available in Supplementary Box S1. The electronic medical files of all eligible patients were evaluated to determine their AF status. In addition, $10 \%$ of those aged $\geq 65$ years were randomly selected using a random number generator and their medical files were scrutinised for AF. The medical history and the use of cardiovascular medication were recorded for patients with newly diagnosed AF and for a $10 \%$ random sample of patients aged $\geq 65$ years. In those with newly diagnosed $A F$, it was additionally noted who had diagnosed the patient and by which pathway, that is, the GP or cardiologist, using the MyDiagnostick, by 12-lead ECG, and/or as a result of suspected symptoms. Screened cases included all patients who filled in informed consent and a short questionnaire on symptoms, and who held the single-lead ECG device. 


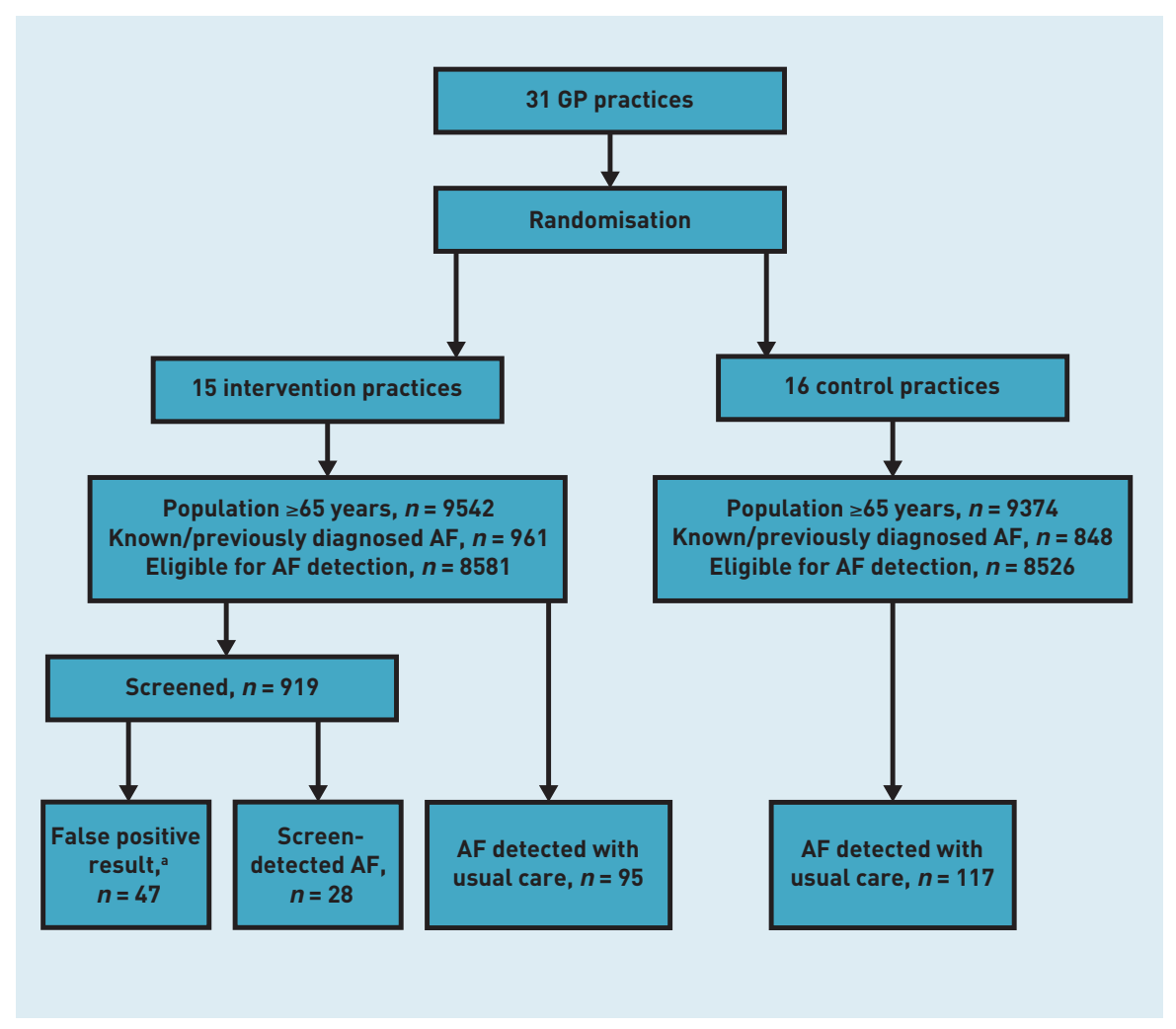

Figure 1. Flow diagram of practice recruitment process. a Patient with false-positive MyDiagnostick result during the study year. Since patients could be screened more than once, the false-positive results can be recorded at a first or a later screening moment. Forty-one of the 47 false positive results were recorded at the first screening. $\boldsymbol{A} \boldsymbol{F}=$ atrial fibrillation.
The study complied with the data protection law of the Netherlands. All participants screened with the MyDiagnostick gave written informed consent. All data relating to patients were anonymised before being sent by the general practice for analyses.

\section{Table 1. Baseline characteristics of the 17107 adults aged $\geq 65$ years with unknown atrial fibrillation status}

\begin{tabular}{|c|c|c|}
\hline Variable & 15 intervention practices & 16 control practices \\
\hline \multicolumn{3}{|l|}{ Patient characteristics } \\
\hline Number of patients, $n$ & 8581 & 8526 \\
\hline Mean age, years (SD) & 74.3 (7.3) & 74.5 (7.3) \\
\hline Females, $n(\%)$ & $4680(54.5)$ & $4610(54.1)$ \\
\hline \multicolumn{3}{|l|}{$\begin{array}{l}\text { Medical history as collected in } \\
\text { a random sample of } 10 \% \text { of both }\end{array}$} \\
\hline \multicolumn{3}{|l|}{ intervention and control group ${ }^{\mathrm{a}}$} \\
\hline Sample size of random samples, $n$ & 867 & 848 \\
\hline \multicolumn{3}{|l|}{ Comorbidities, $n(\%)$} \\
\hline Hypertension & $441(50.9)$ & $427(50.4)$ \\
\hline Type 2 diabetes & $172(19.8)$ & $145(17.1)$ \\
\hline COPD & $70(8.1)$ & $68(8.0)$ \\
\hline Prior myocardial infarction & $59(6.8)$ & $57(6.7)$ \\
\hline Ischaemic stroke ${ }^{b}$ & 34 (3.9) & $54(6.4)$ \\
\hline TIA & $40(4.6)$ & $40(4.7)$ \\
\hline
\end{tabular}

${ }^{a}$ Because of rounding at practice level, sample size is not an exact $10 \%$ in either the intervention or control arm. 'Strokes were defined as either ischaemic or stroke of undefined origin. COPD = chronic obstructive pulmonary disease. SD = standard deviation. $T I A=$ transient ischaemic attack.

\section{Sample size}

For the primary outcome, it was calculated that 10000 people (5000 in each arm) should be included. The effect sizes used for the power calculation were based on a UK study performed in 2002, ${ }^{13}$ in which AF was newly detected in $1.0 \%$ of the control arm lusual care) and $1.6 \%$ in the intervention arm GP practices over 1 year. The intervention arm was composed of two groups: one group who received screening with a single 12-lead ECG and the other with pulse palpation, and $53 \%$ and $69 \%$, respectively, of eligible people aged $\geq 65$ years, were screened. ${ }^{13}$ In the Netherlands, everybody is enlisted with a GP; $90 \%$ of those aged $\geq 65$ years consult the GP practice at least once per year and on average eight times per year. ${ }^{18,19}$ It was therefore assumed that practices would manage to screen $\geq 80 \%$ of these eligible patients over a year because the singlelead ECG device used seemed to be more convenient than pulse palpation or 12-lead ECG. Based on these assumptions, it was estimated that $2.0 \%$ of newly diagnosed cases with AF would be detected in the intervention arm and $1.0 \%$ in the control arm. A 5\% significance level, $90 \%$ power, and an inter-cluster correlation coefficient of 0.0027 were used.

\section{Randomisation}

GP practices were randomised using a random number generator, and cluster size was the number of patients enlisted in each practice.

\section{Data analysis}

Logistic regression analysis was used to compare overall 12 months' incidence rates between arms on an intention to treat' basis. Initially, a random intercept was incorporated into the logistic regression analysis to correct for clustering. Since clustering adjustment showed no or very limited impact of clustering (standard deviation [SD]² close to 0), 'standard' logistic regression was applied. For comparison between the screened and non-screened populations, the two-sided $\chi^{2}$ and Fisher's exact test were used for dichotomous variables and the Student's $t$-test was used for continuous variables.

\section{RESULTS}

In total, 31 GP practices participated, including 18916 enlisted people aged $\geq 65$ years, with 1809 (9.6\%) who had a history of AF at baseline and were excluded from the trial (Figure 1). The study population included 8581 patients in the intervention arm and 8526 in the control 
Table 2. $\mathrm{CHA}_{2} \mathrm{DS}_{2}-\mathrm{VASc}$ score and initiated anticoagulant treatment in patients with newly diagnosed atrial fibrillation

\begin{tabular}{|c|c|c|c|}
\hline \multirow[b]{2}{*}{ Variable } & \multicolumn{2}{|c|}{ Intervention arm } & \multirow{2}{*}{$\begin{array}{c}\text { Control arm } \\
\text { Regularly detected } \\
\text { AF, } N=117\end{array}$} \\
\hline & $\begin{array}{c}\text { Screen-detected } \\
\text { AF, } N=\mathbf{2 8}\end{array}$ & $\begin{array}{l}\text { Regularly detected } \\
\text { AF, } N=95\end{array}$ & \\
\hline Mean $\mathrm{CHA}_{2} \mathrm{DS}_{2}$-VASc score ${ }^{\mathrm{a}}$ (SD) & $3.6(1.6)$ & $4.0(1.5)$ & 3.9 (1.5) \\
\hline $\mathrm{CHA}_{2} \mathrm{DS}_{2}-$ VASc score $1, n(\%)$ & $3(10.7)$ & $6(6.3)$ & $3(2.6)$ \\
\hline Female, $n(\%)$ & $15(53.6)$ & $51(53.7)$ & $71(60.7)$ \\
\hline $\mathrm{CHA}_{2} \mathrm{DS}_{2}$-VASc score 2 in females, $n(\%)$ & $0(0.0)$ & 4 (7.8) & $8(11.3)$ \\
\hline \multicolumn{4}{|l|}{$\begin{array}{l}\text { Initiation of anticoagulant } \\
\text { treatment, }{ }^{\mathrm{b}} n(\%)\end{array}$} \\
\hline VKA & $18(64.3)$ & $45(47.4)$ & $68(58.1)$ \\
\hline NOAC & $5(17.9)$ & $41(43.2)$ & $34(29.1)$ \\
\hline Antiplatelet & $0(0.0)$ & $1(1.1)$ & $4^{c}(3.4)$ \\
\hline \multicolumn{4}{|l|}{$\begin{array}{l}\text { Details about anticoagulant } \\
\text { treatment, } n(\%)\end{array}$} \\
\hline According to guidelines ${ }^{d}$ & $26(92.9)$ & 89 (93.7) & $106(90.6)$ \\
\hline Intended deviation from guidelines ${ }^{e}$ & $1(3.6)$ & $4(4.2)$ & 5 (4.3) \\
\hline Unintended deviation from guidelines ${ }^{f}$ & $1(3.6)$ & $2(2.1)$ & $6(5.1)$ \\
\hline
\end{tabular}

${ }^{a} \mathrm{CHA}_{2} \mathrm{DS} \mathrm{S}_{2}$-VASC = Congestive heart failure (1 point); Hypertension (1 point); Age > 75 years (2 points); Diabetes mellitus (1 point); Stroke including ischaemic stroke or transient ischaemic attack (2 points); Vascular disease including myocardial infarction, angina pectoris, coronary intervention, peripheral artery disease, arterial, or venous thrombosis (1 point); Age 65-75 years (1 point). bInitiation of oral anticoagulant (OAC) treatment: some patients used OAC before diagnoses for mechanical heart valve or venous thromboembolism (VTE) (either lifelong prescription due to multiple VTE or temporary for recent first VTE); this was 2 for AF detection by screening, 4 for detection in usual care of intervention arm, and 4 for detection in control arm. Including two cases in whom either fragmin or clopidogrel was initiated. "Treatment according to the Dutch College of General Practitioners guideline for patients treated in primary care and the European Society of Cardiologists guideline for patients treated in hospital.6.7 ${ }^{e}$ Documented reason for deviation. ${ }^{\prime}$ No documented reason for deviation from guidelines. $A F=$ atrial fibrillation. NOAC $=$ novel oral anticoagulants. $S D=$ standard deviation. $V K A=$ vitamin $K$ antagonists.

arm. The mean age of patients was 74.3 (SD $=7.3$ ) years in the intervention and 74.5 (SD $=7.3$ ) years in the control arm, and comorbidities were equally distributed in both arms (Table 1).

The yield of newly diagnosed AF was comparable in intervention and control practices $(123,1.43 \%$ versus $117,1.37 \%$, $P=0.73$ ) (Figure 1); odds ratio $(\mathrm{OR})=1.05$

Table 3. Medical history of patients (aged $\geq 65$ years) in the intervention GP practices

\begin{tabular}{|c|c|c|c|}
\hline Variable & $\begin{array}{c}\text { Non-screened } \\
\text { population, } N=7662\end{array}$ & $\begin{array}{c}\text { Screened } \\
\text { population, } N=919\end{array}$ & $P$-value ${ }^{a}$ \\
\hline Mean age, years (SD) & $74.3(7.4)$ & $74.8(6.5)$ & 0.057 \\
\hline Female, $n(\%)$ & $4186(54.6)$ & $494(53.8)$ & 0.61 \\
\hline \multicolumn{4}{|c|}{ Sample $\left(N=770^{\mathrm{b}}\right)$ comorbidity, $n(\%)$} \\
\hline Hypertension & $375(48.7)$ & $551(60.0)$ & $<0.001$ \\
\hline Type 2 diabetes & $143(18.6)$ & 223 (24.3) & 0.001 \\
\hline COPD & $57(7.4)$ & 104 (11.3) & 0.003 \\
\hline Prior myocardial infarction & $48(6.2)$ & $78(8.5)$ & 0.025 \\
\hline Ischaemic stroke & 30 (3.9) & 36 (3.9) & 0.98 \\
\hline TIA & $32(4.2)$ & $50(5.4)$ & 0.22 \\
\hline
\end{tabular}

aP-value on difference in non-screened and screened population. ${ }^{b}$ Comorbidity is collected for a random $10 \%$ sample of total population. 'Strokes were defined as either ischaemic or stroke of undefined origin. COPD = chronic obstructive pulmonary disease. $S D=$ standard deviation. $T I A=$ transient ischaemic attack.
(95\% confidence interval $[\mathrm{Cl}]=0.81$ to 1.35$)$. Of the 123 newly detected AF cases in intervention GP practices, 28 (22.8\%) were detected by screening and the remaining 95 $(77.2 \%)$ were detected in usual primary care, for example, by presentation of symptoms or during blood pressure measurement. Mean $\mathrm{CHA}_{2} \mathrm{DS}_{2}$-VASc score was 4.0 for newly detected AF cases in the intervention practices during regular care, 3.9 in control practices, and 3.6 for the 28 screen-detected cases (Table 2).

Intervention practices screened on average $10.7 \%$ of the eligible population (919 of 8581 patients) and found newly detected AF in 28 of these patients $13.0 \%$ of the screened population) (Figure 1). Forty-seven patients (5.1\%) had a false positive MyDiagnostick result (Figure 1). The screened population were a comparable age to the nonscreened population 174.8 years versus 74.3 years), but had greater comorbidity, including hypertension $160.0 \%$ versus $48.7 \%$, $P<0.001)$, type 2 diabetes $(24.3 \%$ versus $18.6 \%, P=0.001)$, and chronic obstructive pulmonary disease (COPD) $(11.3 \%$ versus $7.4 \%, P=0.003$ ) (Table 3).

Approximately half of new AF cases were diagnosed in primary care (intervention 50\% versus control 54\%) (Figure 2). Figure 3 shows for each intervention practice the total rate of newly detected AF and the rate of screen-detected AF as a function of the screened percentage in that practice.

\section{DISCUSSION}

\section{Summary}

In this pragmatic, cluster randomised controlled trial there was no difference between the overall rate of newly detected AF when using a single-lead ECG device for opportunistic screening at the convenience of the general practice compared with usual care (1.43\% versus $1.37 \%, P=0.73)$. In total, 919 patients (10.7\%) were screened in intervention practices and these patients more often had the comorbidities hypertension $160.0 \%$ versus $48.7 \%$ ), type 2 diabetes (24.3\% versus $18.6 \%)$, and/or COPD (11.3\% versus $7.4 \%$ ) than those who were not screened. In the intervention arm, 28 new cases of AF (3.0\% of the screened population) were found by screening, and another 95 new cases of $\mathrm{AF}$ were detected during regular medical care by the GP or hospital specialist.

\section{Strengths and limitations}

This pragmatic trial illustrates how opportunistic screening would be executed in primary care when left to the discretion of the GP practice. The participation rate in the study was low $(10.7 \%)$, and much lower 


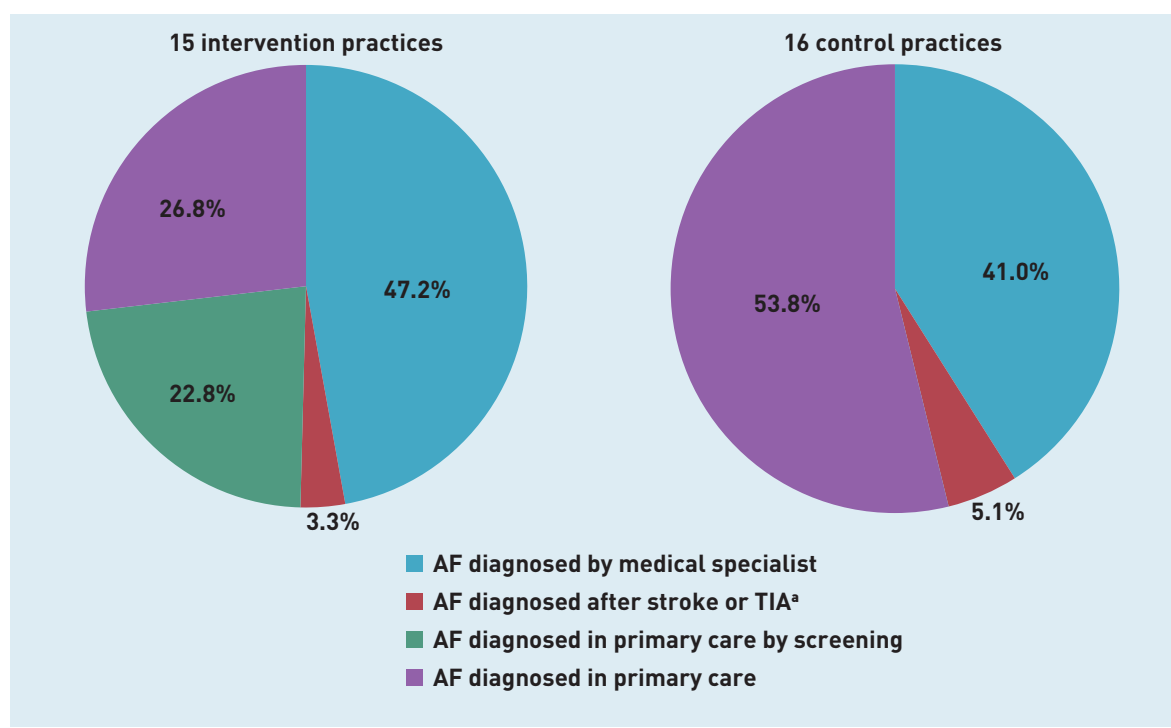

Figure 2. Proportion of cases with atrial fibrillation diagnosed in primary or secondary care Icontrol and intervention), and the proportion diagnosed by screening (intervention).

aStrokes defined as either ischaemic or stroke of undefined origin. $A F=$ atrial fibrillation. $T I A=$ transient ischaemic attack

Figure 3. Percentage of people screened versus rate of all newly diagnosed $A F$ and only screen-detected $A F$ per intervention arm practice. $A F=$ atrial fibrillation. than expected with $80 \%$ used for the power calculation. In the Netherlands, everybody is enlisted with a GP; $90 \%$ of those aged $\geq 65$ years consult the GP practice at least once per year and on average eight times per year. ${ }^{18,19}$ Therefore, potentially $>85 \%$ of the eligible patients could have been screened, even multiple times during a year. The following factors likely played a role in the low participation rate: a lack of financial incentive; 'inertia' of GPs when it comes to screening; time constraints for GPs; the need for written informed consent imposed by the medical ethics committee; and a short questionnaire needing to be filled in. In particular, the need to fill in an informed consent and short questionnaire on symptoms had a disincentive effect and likely resulted in lower numbers of screened patients than would be achieved in real-world implementation (where GPs would ask patients if they would like to be screened without paperwork).

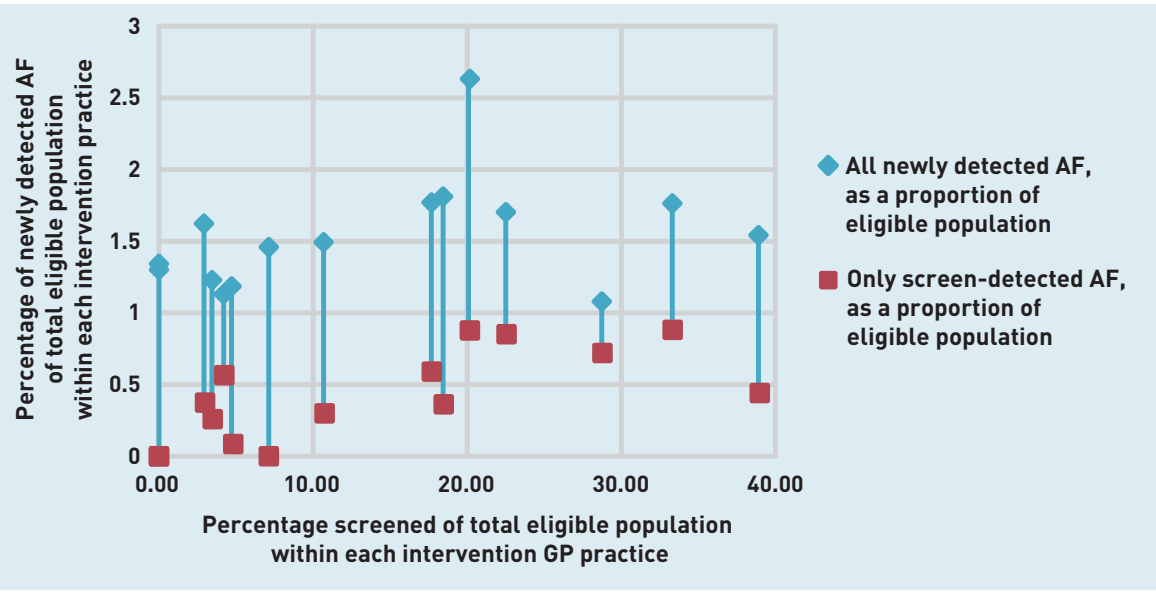

Furthermore, a detection rate of $1.0 \%$ of new AF cases in the control GP practices was assumed based on existing studies, but this was higher in the present study at $1.37 \%$. Even though a 3\% detection rate of $A F$ in the screened population seems promising, it is not possible to determine whether higher participation rates would cause a clinically relevant increased AF detection.

Information from incidental practices reporting showed that some people held the device without completing the survey. They were not counted as screened cases; rather only those who completed the informed consent and short questionnaire were included. It is unclear how many patients this concerned and whether this could have caused selection bias.

The study protocol included as secondary outcome the incidence of cardiovascular events. These numbers were not reported because there was a lack of difference in newly detected AF cases between the arms.

\section{Comparison with existing literature}

The screening rate in the current study was much lower than in the UK primary care study performed in 2002, ${ }^{13}$ in which $53 \%$ of those in the systematic screening arm and $69 \%$ of those in the GP-instructed opportunistic arm were screened. A positive stimulus had been the invitation by letter to people aged $\geq 65$ years to have a 12-lead ECG (systematic arm) and patients' files were flagged to encourage GPs to palpate their pulse when visiting the GP office (opportunistic arm). Another study compared systematic pulse taking by a nurse with opportunistic pulse taking by a doctor or nurse in primary care. ${ }^{12}$ All patients in the systematic arm were sent an explanatory leaflet and an invitation letter to make a specific appointment at the GP surgery. The screening rates were $73 \%$ in the systematic and $29 \%$ in the opportunistic screening arm, clearly demonstrating that 'promotion' is effective for motivating patients and increasing participation rate. ${ }^{12}$ In another study by the authors of the present study, a higher participation rate of $35 \%$ was achieved by combining screening with the influenza vaccination programme in primary care. ${ }^{10}$ Some screening approaches were already successfully implemented in primary care, although with involvement of public health and hospital care, with cervical cancer, colon cancer, and breast cancer achieving uptakes of $61.0 \%, 72.6 \%$, and $78.8 \%$, respectively lfurther information is available from the authors on request). ${ }^{18}$ Importantly, these programmes use a 


\section{Funding}

Femke Kaasenbrood, Monika Hollander, and Frans $H$ Rutten received an unrestricted institutional grant from Boehringer Ingelheim to conduct this study lgrant reference number: S1357832528). All researchers that contributed to this trial were independent from funders (ClinicalTrials.gov Identifier: NCT02270151).

\section{Ethical approval}

The Medical Ethics Committee (METC) of the University Medical Center Utrecht confirmed that the Medical Research Involving Human Subjects Act (WMO) does not apply to this trial (METC-protocol number 14-163/C).

\section{Provenance}

Freely submitted; externally peer reviewed.

\section{Competing interests}

Robert G Tieleman has received grants and personal fees from Boehringer Ingelheim, and personal fees from Pfizer/BristolMyers Squibb and Daiichi Sankyo, outside of the present study. Robert G Tieleman has a patent filled by Applied Biomedical Systems with royalties paid. Robert $G$ Tieleman receives no personal payment from any industrial partner. Arno $W$ Hoes chairs a large research and teaching institute at the University Medical Center Utrecht that performs investigator- and industrydriven research projects with a number of pharmaceutical and diagnostic companies. In addition, some of Arno W Hoes's staff members receive unrestricted grants for research projects from a number of companies. It is the authors' explicit policy to work with several companies and not to focus on one or two industrial partners. The other authors have declared no competing interests.

\section{Acknowledgements}

The authors would like to thank all participating patients and GP practices for their contribution to this study.

\section{Discuss this article}

Contribute and read comments about this article: bjgp.org/letters systematic approach with direct individual patient invitation; receive substantial governmental incentives; and have large public media campaigns on awareness among the general public.

There are important implementation barriers to screening for $A F$ in primary care. These include a lack of prompts in the regular electronic medical files; a lack of financial incentive; 'inertia' of GPs when it comes to screening; being used to acting on a patient's symptom presentation; and putting an additional task into an already high workload. ${ }^{20}$ These barriers are not easy to overcome but options to be considered are systematic organisation of screening, for example, during influenza vaccination sessions; combining AF screening with existing primary care disease management programmes, for example, patients with type 2 diabetes or cardiovascular risk management, preferably going along with sufficient financial incentives; and the use of prompts in the patient's electronic medical file.

The lack of difference between intervention and control practices in the current study might also be due to the high prevalence of already known AF: $9.6 \%$ of those aged $\geq 65$ years. This is substantially higher than $7.2 \%$ in the previously mentioned UK study. ${ }^{13}$ In line with this, the detection rate of new AF with usual care in the control practices was $40 \%$ higher than in usual care in the UK study $(1.4 \%$ versus $1.0 \%) .{ }^{13}$ Both findings suggest that $A F$ is currently better detected by usual primary care than it was a decade ago. The increased attention of GPs to AF over the last decade might have increased their awareness about screening for $A F$ in older patients and highrisk groups, and in patients with symptoms such as palpitations or shortness of breath. Intervention practices mainly screened patients with comorbidities, which might have led to a higher screen-detection rate 13.0\% of all screened patients) compared with previous primary care screening studies (on average 1.4\%). ${ }^{11}$ It remains unknown whether these patients would also have been detected with usual care.

\section{Implications for research}

Opportunistic screening with a single-lead ECG device at the discretion of the GP seems not to be effective. Further research should investigate whether systematic screening for AF with a higher participation rate increases detection yield in contemporary usual care and how screening should be organised. Further research is also needed into the barriers and facilitators to improve screening for AF in primary care. 


\section{REFERENCES}

1. Heeringa J, van der Kuip DAM, Hofman A, et al. Prevalence, incidence and lifetime risk of atrial fibrillation: the Rotterdam study. Eur Heart J 2006; 27(8): 949-953.

2. Benjamin EJ, Wolf PA, D'Agostino RB, et al. Impact of atrial fibrillation on the risk of death: the Framingham Heart Study. Circulation 1998; 98(10): 946-952.

3. Wolf PA, Abbott RD, Kannel WB. Atrial fibrillation as an independent risk factor for stroke: the Framingham Study. Stroke 1991; 22(8): 983-988.

4. Hart RG, Pearce LA, Aguilar MI. Meta-analysis: antithrombotic therapy to prevent stroke in patients who have nonvalvular atrial fibrillation. Ann Intern Med 2007; 146(12): 857-867.

5. Rienstra M, Vermond RA, Crijns HJGM, et al. Asymptomatic persistent atrial fibrillation and outcome: results of the RACE study. Heart Rhythm 2014; 11(6): 939-945

6. Dutch College of General Practitioners Guideline Development Group for Atrial fibrillation. Guideline Atrial fibrillation (second partial revision). [In Dutch]. Huisarts Wet 2013; 56: 392-401. https://uww.nhg.org/standaarden/ samenvatting/atriumfibrilleren (accessed 7 Jan 2020).

7. Kirchhof P, Benussi S, Kotecha D, et al. 2016 ESC Guidelines for the management of atrial fibrillation developed in collaboration with EACTS Europace 2016; 18(11): 1609-1678.

8. Taggar JS, Coleman T, Lewis S, et al. Accuracy of methods for detecting an irregular pulse and suspected atrial fibrillation: a systematic review and metaanalysis. Eur J Prev Cardiol 2016; 23(12): 1330-1338.

9. Engdahl J, Andersson L, Mirskaya M, Rosenqvist M. Stepwise screening of atrial fibrillation in a 75-year-old population: implications for stroke prevention. Circulation 2013; 127(8): 930-937.

10. Kaasenbrood F, Hollander M, Rutten FH, et al. Yield of screening for atrial fibrillation in primary care with a hand-held, single-lead electrocardiogram device during influenza vaccination. Europace 2016; 18(10): 1514-1520.
11. Lowres N, Neubeck L, Redfern J, Freedman SB. Screening to identify unknown atrial fibrillation. A systematic review. Thromb Haemost 2013; 110(2): 213-222.

12. Morgan S, Mant D. Randomised trial of two approaches to screening for atrial fibrillation in UK general practice. Br J Gen Pract 2002; 52(478): 373-380.

13. Fitzmaurice DA, Hobbs FDR, Jowett S, et al. Screening versus routine practice in detection of atrial fibrillation in patients aged 65 or over: cluster randomised controlled trial. BMJ 2007; 335(7616): 383.

14. Tieleman RG, Plantinga Y, Rinkes D, et al. Validation and clinical use of a novel diagnostic device for screening of atrial fibrillation. Europace 2014; 16(9): $1291-1295$

15. Vaes B, Stalpaert S, Tavernier K, et al. The diagnostic accuracy of the MyDiagnostick to detect atrial fibrillation in primary care. BMC Fam Pract 2014; 15: 113

16. Kowey PR, Stebbins D, Igidbashian L, et al. Clinical outcome of patients who develop PAF after CABG surgery. Pacing Clin Electrophysiol 2001; 24(2): 191193

17. Maisel WH, Rawn JD, Stevenson WG. Atrial fibrillation after cardiac surgery Ann Intern Med 2001; 135(12): 1061-1073.

18. Statline. Contacts registered by the doctor; age and gender. 2019. https:// opendata.cbs.n//statline/\#/CBS/nl/dataset/80191NED/table?fromstatweb (accessed 6 Jan 2020).

19. Statline. Population on January 1 and average; gender, age and region. 2019. https://opendata.cbs.n//statline/\#/CBS/nl/dataset/03759ned/table?fromstatweb laccessed 6 Jan 2020)

19. Orchard J, Neubeck L. Freedman B, et al. eHealth tools to provide structured assistance for atrial fibrillation screening, management, and guidelinerecommended therapy in metropolitan general practice: the AF - SMART Study. J Am Heart Assoc 2019; 8(1): e010959.

20. Sanmartín M, Fraga FF, Martín-Santos A, et al. A campaign for information and diagnosis of atrial fibrillation: 'pulse week'. Rev Esp Cardiol (Engl Ed) 2013; 66(1): 34-38. 